\title{
Development of mathematical model of Universal modeling method for centrifugal compressors calculation
}

\author{
Yuri Galerkin ${ }^{1}$, Aleksandr Drozdov ${ }^{1, *}$, Olga Solovyeva ${ }^{1}$, and Kirill Kabalyk ${ }^{2}$ \\ ${ }^{1}$ Peter the Great St. Petersburg Polytechnic University, Polytechnicheskaya 29, Saint Petersburg, 195251 Russia \\ ${ }^{2}$ Lodz University of Technology, Institute of Turbomachinery Wolczanska 219/223 Lodz, Poland 90-924
}

\begin{abstract}
The heart of the Universal modeling engineering method is the physical model that is based on flow visualization and measurements inside rotating impellers. The experience of its application allowed finding ways of mathematical model development. The loss of a head is a function of flow gradients along a surface and along a normal to it. Necessary calculations were 1-D in the previous models. Quasi-threedimensional approach is applied in the new model. A new loading factor model was applied. It determines characteristics by the angle of inclination and the value of the loading factor at zero flow rate. This made it possible to abandon the choice of empirical coefficients. A new loss of head model in a vaneless diffuser based on the results of CFD calculations generalization is used. The new model allowed abandoning a number of empirical coefficients of the mathematical models. Identification of the new mathematical model by the characteristics of model tests of centrifugal compressor stages was made. The efficiency calculation accuracy was sufficient for the engineering method.
\end{abstract}

\section{Introduction}

The TU SPb R \& D laboratory of compressor problems was active in $1960-1990$ in researches of turbo compressor gas dynamics. Measurements inside rotating impellers, different visualization technologies, etc. lead to advanced physical and math models development processes and phenomena occurring in them for decades, as well as designing centrifugal compressors for different industries [1 - 11]. The math model and the PC programs named the Universal modeling method were developed and widely applied in design practice [11]. The $\mathrm{R} \& \mathrm{D}$ centers everywhere work on the same problems [12 - 28].

The programs of the Universal modeling method (UMM) are widely used in the practice of calculation and design of centrifugal compressors and compressor stages. Programs have proved their high efficiency. A new generation of centrifugal superchargers and exchangeable flow paths for the gas industry was created by these programs [29]. Taking into account all the projects implemented by SPbPU, in the industry of the Russian Federation and other countries in 2010 there were about 430 centrifugal compressors and exchangeable flow paths of more than fifty types with a total installed capacity of more than 5 million $\mathrm{kW}$ [30].

The basis of the UMM mathematical model is a physical model of gas flow in the flow path of the centrifugal compressor stage. The efficiency and work coefficient depend on the flow path form, the similarity criteria (isoentropic coefficient $k$, relative Mach number $M_{u}$, relative Reynolds number $\mathrm{Re}_{u}$ ), relative flow rate coefficient. To determine the efficiency of the flow path, head losses in each of its elements are calculated, which are determined by formulas involving empirical coefficients $X(i)$.

Description of the mathematical model is presented in [31, 32]. Researches in the R\&D laboratory "Gas dynamics of turbomachines" allowed to accumulate the results that leaded in the creation of the 9th version of the Universal modeling method. The main features of this model are presented below.

\section{Methods}

\subsection{The quasi-three-dimensional approach to the calculation of 3D impellers}

A significant unevenness of the flow along the 3D impeller blades height leads to the fact that the use of a $1 \mathrm{D}$ approach to the calculation of losses gives errors. The displacement of the calculated current line from the midline closer to the periphery, proposed in [33] does not fully solve this problem. The most effective is the a quasi-three-dimensional approach to the calculation of losses in the 3D impellers blade rows.

\footnotetext{
* Corresponding author: a drozdi@mail.ru
} 
For this purpose, in the 9th version of the mathematical model, the flow in the blade row is divided into 8 current lines, for each of which incidence losses, friction losses on the blades and mixing losses are calculated. Friction losses on the hub and shroud surfaces take place on the 1st and 8th current lines. Mixing losses in case VLD width is greater than the 3D impeller blade height at the output $\zeta_{\text {mixb3/b2 }}$ are also considered in a one-dimensional formulation.

At the blade row inlet it is assumed that the flow coefficient $\varphi_{1 i}^{\prime}$ is the uniform at each current line. Relative velocity at the blade row inlet:

$$
\bar{w}_{1 i}^{\prime}=\sqrt{\bar{D}_{1 i}^{2}+\varphi_{1 i}^{\prime 2}},
$$

where $\bar{D}_{1 i}$ is relative impeller inlet diameter.

Inlet flow angle and other parameters are calculated on each current line. Next, the calculation algorithm for each current line repeats the $1 \mathrm{D}$ calculation of the 8th version.

The blades height at the output is relatively small and the unevenness of the flow along its height can be neglected. In section "2" the calculation returns to the $1 \mathrm{D}$ approach. The polytrophic coefficient of the process between sections "1" and "2" is calculated based on the amount of losses on each current line:

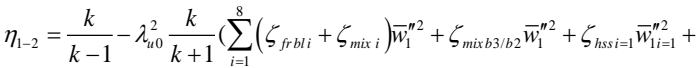

$$
\begin{aligned}
& \left.+\zeta_{\text {hsi } i=8} \bar{W}_{i=8}^{\prime \prime 2}+\sum_{i=1}^{8}\left(\zeta_{i n i} \bar{W}_{1 i}^{\prime \prime 2}\right)+2 \psi_{T}\left(\beta_{l k}+\beta_{f r}\right)\right) /\left[\frac{T_{1}^{\prime}}{T_{0}^{*}}\left(\frac{T_{2}}{T_{1}^{\prime}}-1\right)\right]
\end{aligned}
$$

where $\eta$ is polytrophic efficiency; $\bar{w}^{\prime \prime}$ is relative velocity in an impeller throat; $\beta_{\text {leac }}$ is labyrinth seal leakage coefficient; ${ }^{\beta}$ fr is disc friction coefficient; $T^{*}$ is total temperature; $T$ is static temperature; $\psi_{T}$ is loading factor; $\lambda$ is velocity coefficient.

\subsection{Model for non-incidence inlet flow rate definition}

Determination the non-incidence flow of impeller blades leading edge is necessary for the calculation of gasdynamic characteristics by UMM. In this flow rate, the direction of the critical streamline (streamline falling on the blade leading edge) coincides with the inlet blade angle. The direction of the critical streamline is affected by the final thickness of the blades, as well as the pressure difference on the pressure and suction blade sides (figure 1).

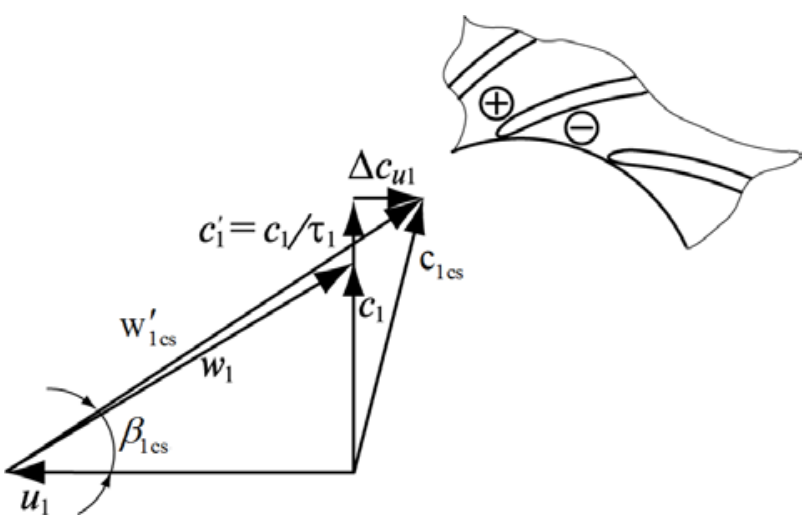

Fig.1. The inlet velocity triangle of the critical streamline [2]

To calculate adjustment amount $\Delta \mathrm{c}_{\mathrm{u} 1}$ in a dimensionless form in Universal modelling method, the following formula is proposed [2]:

$$
\Delta \overline{\mathrm{c}}_{\mathrm{u} 1}=\frac{\psi_{\mathrm{T}}}{\mathrm{z}\left(1-\mathrm{K}_{\mathrm{pd}}\right)\left(1-\overline{\mathrm{D}}_{1}\right)} \sin \beta_{\mathrm{bl1}},
$$

where $z$ is number of blades; $\beta_{b l 1}$ is blade angle to tangential direction; $K_{p d}$ is empirical coefficient of velocity diagram.

The results of calculation by the UMM mathematical model checked by inviscid Q3D program 3DM.023. Researches of the compressor engineering problem laboratory LPI [3] showed a good agreement between the measured and calculated by 3DM.023 velocity diagrams. The comparison of non-incidence modes of UMM and Q3D inviscid flow showed good agreement for high flow rate stages and serious difference of about $26 \%$ for low flow rate of stages (a mathematical model has shifted design mode to the right). These results led to the necessity of reviewing and adjusting the nonincidence flow model.

Fig. 1 not quite correctly defines the interaction betwixt flow and vortex. The effect of the vortex with a circulation on the flow near a leading edge in a more rigorous formulation is illustrated in Fig. 2.

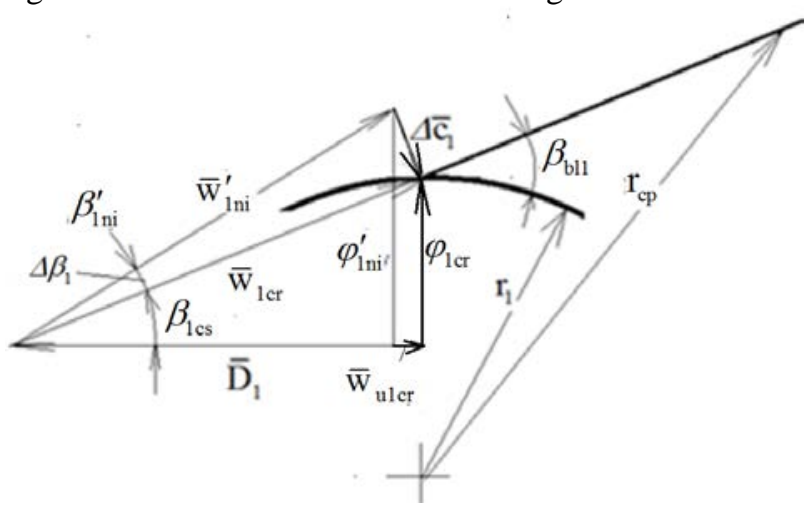

Fig.2. Scheme of the action of the vortex replacing the vane on the flow near a leading edge

The vortex located on a radius $r_{\mathrm{cp}}$ (Fig. 2) induces a velocity component $\Delta \overline{\mathrm{c}}_{1}$ that is perpendicular to the direction from the vortex to the leading edge. In the corrected model the next simplifying assumptions are made: 
- $\Delta \overline{\mathrm{C}}_{1}$ is perpendicular to the direction of the inlet blade angle;

- the distance to the leading edge from the pressure center is equal to the difference in radii divided by the sinus of the inlet blade angle.

Taking into account the relations in Fig. 2 under the accepted conditions:

$$
\Delta \overline{\mathrm{c}}_{1}=\frac{\psi_{\mathrm{T}}}{\mathrm{z}} \frac{\sin \beta_{\mathrm{bl1}}}{\left(1-\mathrm{K}_{\mathrm{pd}}\right)\left(1-\overline{\mathrm{D}}_{1}\right)} .
$$

Applying the equation (4) not bring the nonincidence mode to calculations by program 3DM.023. However, the new scheme is more accurate than previous. To account for differences in the shape and size of the rows blade, an empirical coefficient $\mathrm{X}$ is applied in equation (4).

Non-incidence flow rate coefficients $\Phi_{\text {ni }}$ were calculated by equation (4) for 20 model stages with 3D impellers, for 6 stages with 2D impellers with blades of a special profile with controlled load, and for more 6 impellers with 2D blades with the mean line as an arc. The analysis of the empirical coefficient dependence on the gap-chord $\overline{\mathrm{t}}_{1}^{\prime}=\frac{\pi \overline{\mathrm{D}}_{1}}{\mathrm{z}} \tau_{1}$ (where $\tau$ is blade blockage factor; $\overline{\mathrm{t}_{1}^{\prime}}$ is relative pitch at an impeller inlet), blade non-dimensional load $\Delta \overline{\mathrm{w}}=\overline{\mathrm{w}}_{\mathrm{s}}-\overline{\mathrm{w}}_{\mathrm{p}}$ (where $\overline{\mathrm{w}}_{\mathrm{p}}$ is relative velocity on pressure side; $\overline{\mathrm{w}}_{\mathrm{s}}$ is relative velocity on suction side), non-dimensional throat area $\overline{\mathrm{a}}_{1}=\overline{\mathrm{t}}_{1}^{\prime} \times \sin \beta_{\mathrm{bl} 1}=\frac{\pi \overline{\mathrm{D}}_{1}}{\mathrm{z}} \tau_{1} \times \sin \beta_{\mathrm{bl} 1}$ was carried out.

As a result, the following formulas are recommended for the empirical coefficient $\mathrm{X}$ :

- 2D impellers with arc blades:

$$
\mathrm{X}=1.37=\text { const . }
$$

- 3D impellers:

$$
\mathrm{X}=1+9.75 \Delta \overline{\mathrm{w}}-31.9 \overline{\mathrm{a}}_{1} .
$$

- 2D impellers with profiled blades:

$$
\mathrm{X}=1+5 \Delta \overline{\mathrm{w}}-24.9 \overline{\mathrm{a}}_{1} \text {. }
$$

The ratio $\Phi_{\text {nimm }} / \Phi_{\text {ni } \mathrm{DM}}$ calculated by eq. (5-7) for 32 impellers is shown in Fig. 3.

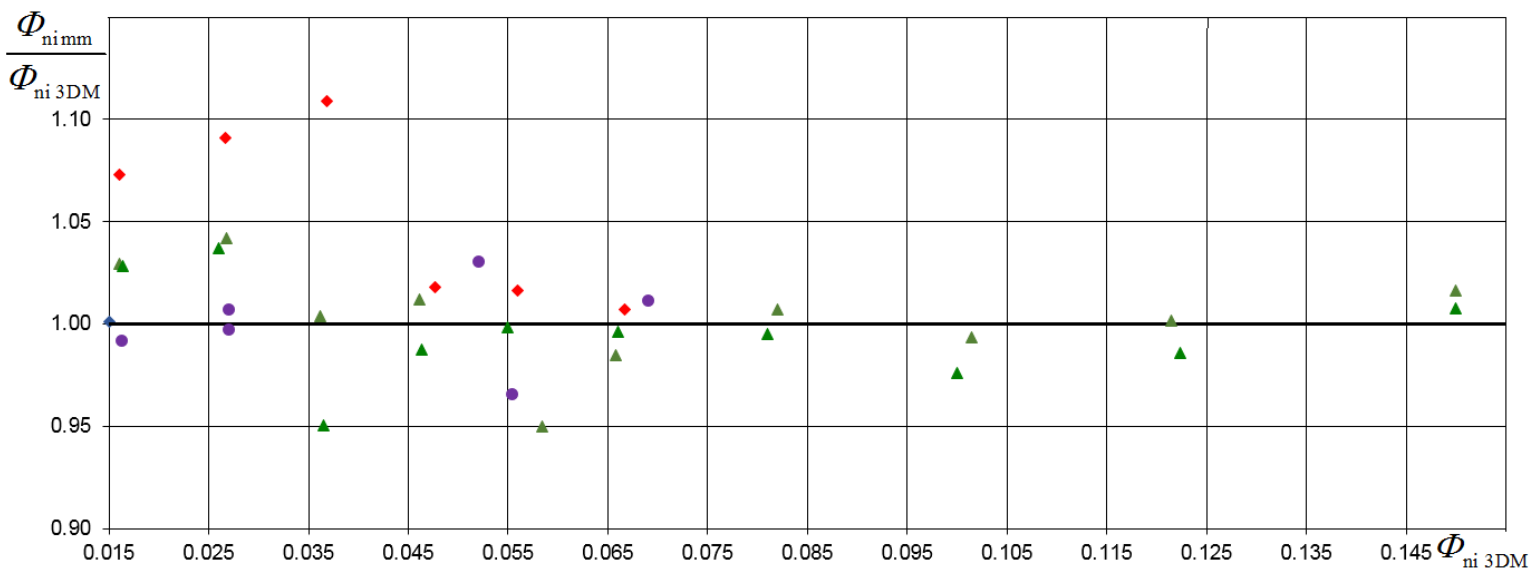

Fig.3. Comparison of non-incidence flow rates calculated by eq. (5 - 7) $\Phi_{\text {ni mm }}$ and by inviscid calculation $\Phi_{\text {ni 3DM }}$ Red - 2D impellers with profiled blades; violet - 2D impellers with ark blades; green - 3D impellers

The average error of calculating the conditional flow coefficient for 20 stages with the 3D impeller was $1.8 \%$, for 7 stages with a 2D with profiled blades - 4.5\%, for stages with 2D with arc blades - 1.6\%. The modeling of 2D impellers with profiled blades is less accurate, but is acceptable for the primary design.

\subsection{A mathematical model of the centrifugal compressor stages loading factor characteristics}

Simulation of loading factor characteristics is an important part in the calculation and design of centrifugal compressor stages. For modeling the loading factor characteristics is still relevant proposed by the V.F. Ris [34] schematization of the power transmission process.

In the mathematical model of the Universal modeling method, the impact of the blade on the flow is replaced by the action of a vortex with the velocity circulation on the blade:

$$
\psi_{T}=\frac{1-\varphi_{2}^{\prime} c t g \beta_{b l 2}}{1+K_{\mu} \frac{1}{z\left(1-\bar{D}_{1}\right) K_{p d ~ d e s}} \sin \frac{\beta_{b l 1}+\beta_{b l 2}}{2}},
$$

where $K_{\mu}$ is empirical coefficient (viscosity influence on a loading factor).

For the second parameter, which allows to model the loading factor characteristic, the pressure coefficient at zero flow rate $\psi_{T 0}$ the formula is used:

$$
\psi_{T 0}=1-X_{\psi_{T 0}} \frac{\Delta \bar{c}_{u 2 d e s}}{2}
$$

A wide range of empirical coefficients variation $K_{\mu}$ and $X_{\psi_{T 0}}$ creates certain difficulties in the calculation and design of new compressor stages. To solve this problem , studies were carried out and a scheme for modeling the loading factor characteristic through its inclination angle $\beta_{T}^{0}$ was proposed. For the $\psi_{T 0}$ an approximating formula without the use of an empirical coefficient was proposed. Steps of work in this direction 
are presented in $[35,36]$. The results had practical application, but needed to be improved and corrected.

At the last stage of approximation the experimental data of 20C family 8 model stages and one compressor participated. The following formula for the approximation of the loading factor inclination angle is obtained:

$$
\beta_{T}^{0}=10.9655\left(\beta_{b l 2}^{0}\right)^{2}+17.4802\left(\frac{l}{t}\right)^{0,4}+18.22 \bar{b}_{2}^{0.5}-5.702\left(\frac{b_{2}}{b_{1}}\right)^{0,8} \text {, }
$$

where $b$ is blade height.
The approximation accuracy is 98.96\%. A comparison of the calculated and experimental data is shown in figure 4.

For the value $\psi_{T 0}$ is obtained formula:

$$
\psi_{T 0}=1-0.0479\left(\frac{l}{t}\right)^{0,9}-0.0025\left(\frac{b_{2}}{b_{1}}\right)^{2}+0.0255\left(\frac{\beta_{b l 2}}{40}\right)^{0,7}-3.7462 \bar{b}_{2}^{1,5},
$$

where $l$ is blade length.

The approximation accuracy is $88.62 \%$. A comparison of the calculated and experimental data is shown in figure 5.

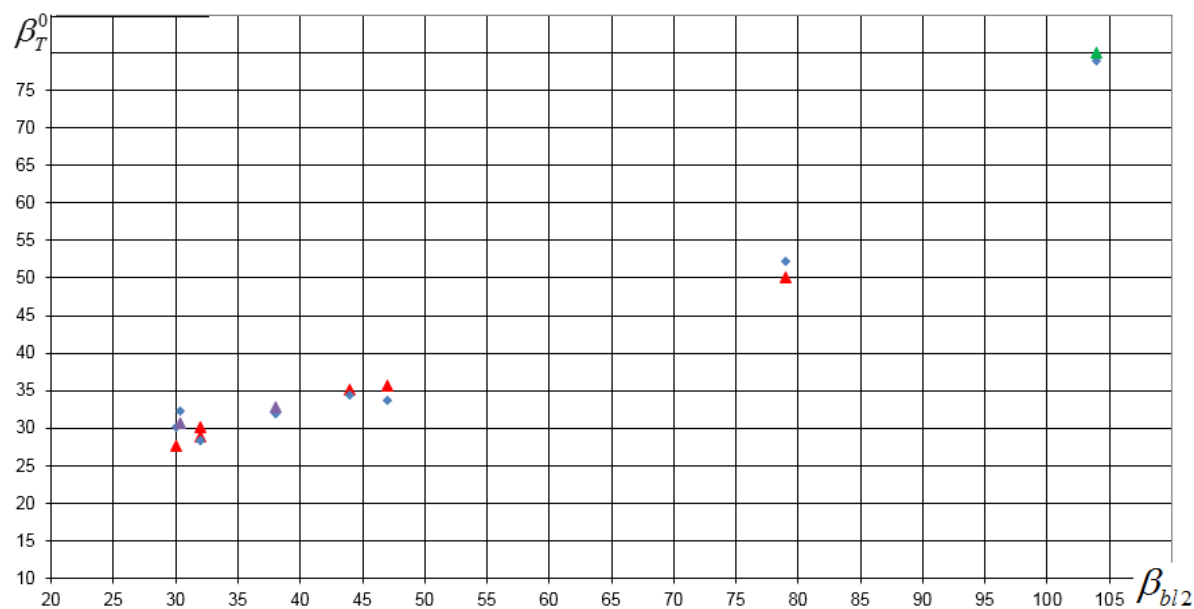

Fig.4. Comparison of calculated by the formula (14) and experimental $\beta_{T}^{0}$ green - compressor; blue - approximation; violet - 2D impellers with arc blades; red - 2D impellers with profiled blades.

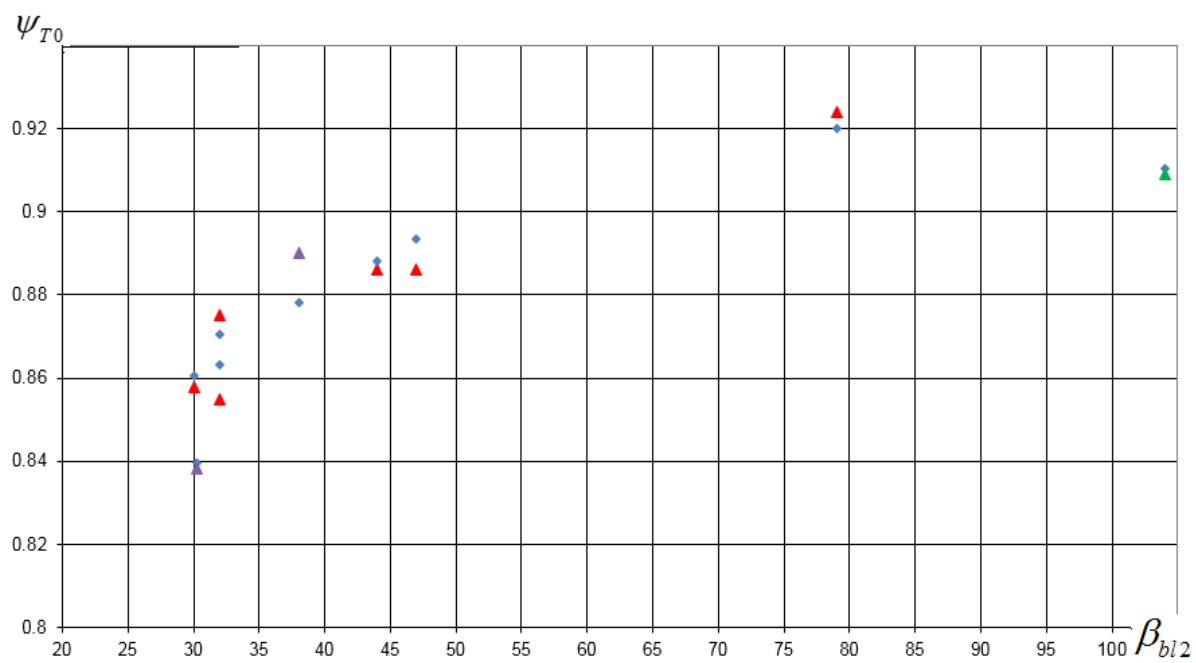

Fig.5. Comparison of calculated by the formula (14) and experimental $\psi_{T 0}$ green - compressor; blue - approximation; violet - 2D impellers with arc blades; red - 2D impellers with profiled blades.

The obtained formulas showed good approximation accuracy and turned out to be simpler than those presented in [35].

\subsection{A mathematical model of head loss in VLD}

The mathematical model of losses in VLD applied in 4-8 versions of UMM [2, 11, 37] assumes division of a diffuser on 20 sections on length. For each of them is calculated the loss ratio of the site associated with the friction coefficient arising from the lost power balance:

$$
\zeta_{\text {sec }}=\frac{\mathrm{c}_{\mathrm{w}} \overline{\mathrm{c}}_{1 \mathrm{sec}}^{2}\left(\overline{\mathrm{D}}_{2 \mathrm{sec}}-\overline{\mathrm{D}}_{1 \mathrm{sec}}\right)}{2 \overline{\mathrm{b}} \sin \alpha_{1 \mathrm{sec}} 0,25\left(\overline{\mathrm{c}}_{1 \mathrm{sec}}^{2}+\overline{\mathrm{c}}_{2 \mathrm{sec}}^{2}\right)} \mathrm{K}_{\lambda} \mathrm{K}_{\mathrm{spat}} \mathrm{K}_{\mathrm{nu}},
$$

where $c_{w}$ is resistance force ratio; $K_{\lambda}$ is correction coefficient (influence of compressibility); $K_{\text {spat }}$ is correction coefficient (influence of the 3D character of the flow); $\mathrm{c}$ is absolute flow velocity; $\alpha_{1 \text { sec }}$ is flow angle with respect to tangent;

The use of CFD techniques makes it possible not less correct, but more simple modeling of VLD. In [38, 39] 
the results of flow calculations and gas - dynamic characteristics of VLD with relative width $\mathrm{b} / \mathrm{D}_{2}=$ $0.014-0.100$, radial length $\mathrm{D}_{4} / \mathrm{D}_{2}$ up to 2.0, in the range of inlet flow angles $\alpha_{2}=10^{0}-90^{0}$ are presented. Similarity criteria varied within $\lambda_{c 2}=0.23-0.82$, $\mathrm{Re}_{b 2}=53$ 000-1 030 000. Comparison with the theory showed the regularity of the gas-dynamic characteristics, and with known experiments - a good coincidence in the flow structure.

The paper [40] presents the process of approximating the VLD loss coefficient and the outlet flow angle and the obtained formulas of the mathematical model:

$$
\zeta, \alpha_{4}=f\left(\bar{b}_{2}, \bar{D}_{4}, \alpha_{2}, \lambda_{c 2}, \operatorname{Re}_{b 2}, \bar{\kappa}_{r g}\right),
$$

where $\bar{\kappa}_{r g}$ is relative surface roughness.

The advantage of the new model is the absence of empirical coefficients that requiring identification. The entire diffuser but not its 20 sectors is calculated. Another advantage is the elimination of the need for iterative equations solution. A new mathematical model provides the possibility of calculation of the surfaces ratio $\mathrm{f}_{4} / \mathrm{f}_{2}=\mathrm{D}_{4} / \mathrm{D}_{2} \times \sin \alpha_{4} / \sin \alpha_{2}$, gas-dynamic functions of the current density [41] can be applied for flow parameters calculation.

\section{Results}

The developed mathematical model needs to be identified. Identification means the selection of the coefficient values that provides the maximum agreement between calculated and experimental efficiency of the modeling stages involved in the identification. To solve this problem R\&D laboratory "Gas dynamics of turbomachines" has a specialized program IDENT. Its database contains information about more than two hundred tests of model stages, the flow path size, the tests conditions and the results.

The identification involved the test results of 30 stages ( 9 of them with LD, the rest with VLD). Basic design parameters range of model stages: $\Phi_{\text {des }}=0.028-$ 0.080, $\quad \psi_{\text {Tdes }}=0.45-0.65, \quad \bar{D}_{h}=0.25-0.373$, $\bar{D}_{4}=1.428-1.615$, $M_{u}=0.60-0.86$, $\operatorname{Re}_{u}=4.8 \times 10^{6}-6.9 \times 10^{6}$. The stages cover a wide range of flow rates and loading factors (figure 6).

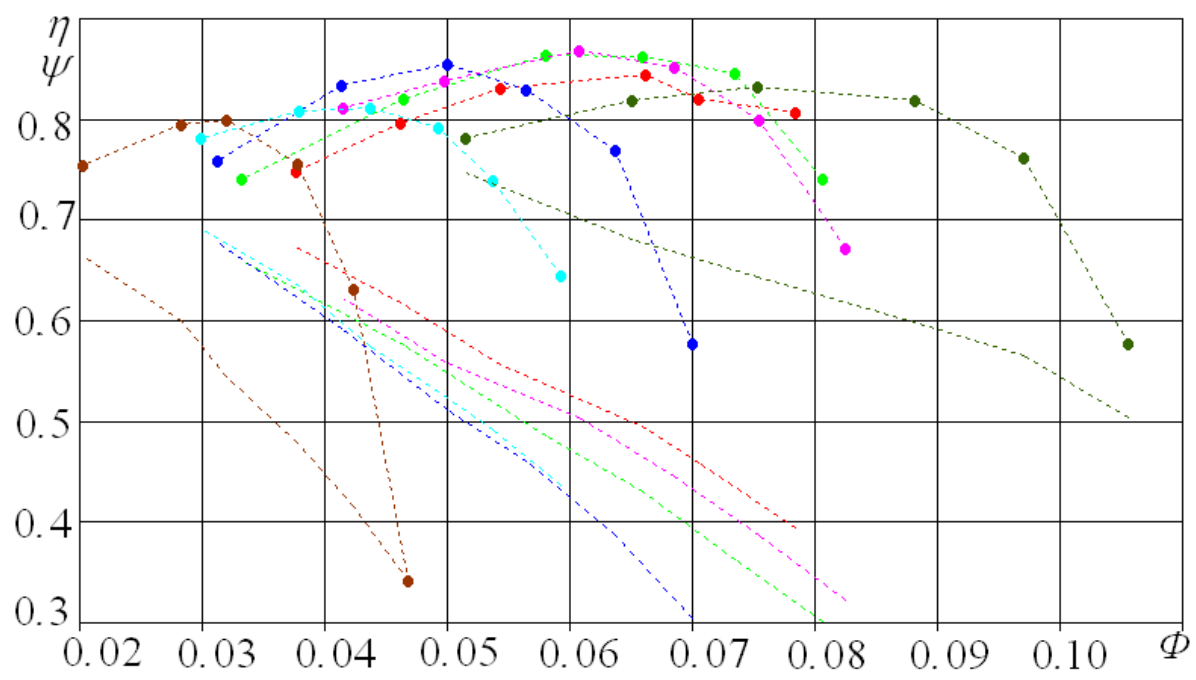

Fig.6. Characteristics of some stages involved in the mathematical model identification

The identification process was carried out by searching the values of empirical coefficients by the comparison calculated and experimental efficiency. Values of coefficients $X(i)$ provided minimal average error of efficiency calculation were selected [2]. In the process of identification, it is also important to control the ratio of the losses components in the flow path elements and between the various elements. This is necessary to obtain a correct, physically based distribution of the loss coefficients for the flow part (losses in the impeller, diffuser, return channel).

The set of empirical coefficients obtained in the [33] is taken as the initial version.

On the first step, the values of empirical coefficients affecting the amount of head losses in all modes (friction losses on the bounding surfaces and blades, etc.) are determined. Identification of these empirical coefficients is made on experimental data for the flow rate with the maximum efficiency. It is assumed that in this mode, the impact of incidence losses is insignificant.

Identification showed the following results: the average calculation error of the design (maximum) efficiency is $0.93 \%$.

After exhausting the possibilities to improve the accuracy of determining the losses in the design flow rate, the identification of empirical coefficients responsible for incidence losses was carried out. It was made separately for the right and left parts of the characteristic. The calculation error for five points (except the highest flow rate) is $1.36 \%$. 


\section{Conclusion}

The changes made in the 9th version of the mathematical model and the corresponding computer programs allowed to increase the performance, accuracy, simplify the user's work.

The obtained mathematical model based on the identification results showed good accuracy of modeling centrifugal compressor stages gas - dynamic characteristics $(0.93 \%$ - for the design flow rate and $1.36 \%$ - for the entire characteristic).

\section{Acknowledgements}

The research was performed by a grant of Russian Science Foundation (project No. 18-79-10165).

\section{References}

1. Y. Galerkin, Compr. Techn.and Pneum. 2, 9-14 (2000)

2. Yu. B. Galerkin, Turbocompressors 650 (2010)

3. Y. Galerkin, Proceedings of compressor scientific school of the SPbSPU, 670 (2010)

4. K. P. Seleznev, Yu. B. Galerkin, Centrifugal compressors 271 (1982)

5. L. N. Marenina, Compr. Techn.and Pneum. 3, 2735 (2016)

6. A. Rekstin, E. Popova, A. Ucehovscy, Oil and Gas Engineering 2007 (2018)

7. A.I. Borovkov, I.B. Voinov, A.F. Rekstin, St. Petersburg Pol. Un. Journ. of Eng. Sc. and Tech. 25 (2), 87-104 (2019)

8. V.B. Semenovskiy, St. Petersburg Pol. Un. Journ. of Eng. Sc. and Tech. 24 (4), 11-23 (2018)

9. A.F. Rekstin, B.V. Bakaev, St. Petersburg Pol. Un. Journ. of Eng. Sc. and Tech. 24 (4) (2018)

10. K. Soldatova, International Conference on Compressors and their Systems (2017)

11. Y. Galerkin, A. Rekstin, K. Soldatova, A. Drozdov, Y.A. Popov, 17 international scientific and technical conference on compressors, 19-29 (2017)

12. D. Japikse, JSME fluid engineering conference, 3444 (2003)

13. D. Japikse, JANNAF Conference (1996)

14. D. Japikse, JANNAF Conference (1998)

15. D. Japikse, M. J. Platt, 10th International Symposium on Transport Phenomena and Dynamics of Rotating Machinery (2004)

16. R.H. Aungier, Centrifugal Compressors: A Strategy for Aerodynamic Design and Analysis (2000)

17. R.H. Aungier, Transactions of the ASME 117, (1995)
18. M. Casey, C. Robinson, Journal of Turbomachinery, 135 (2013)

19. A. T. Lunev, Compr. Techn. and Pneum. 10, 4-7 (2001)

20. A. N. Lyubimov, V. E. Evdokimov, Compr. Techn.and Pneum. 7, 28-33 (2012)

21. J. Schiff, Master's thesis, 171 (2013)

22. A.Y. Procofiev, PhD thesis, 190 (2003)

23. D. Barsi, A. Perrone, Y. Qu, V. Sergeev, P. Zunino, Journ. of Therm. Sc. 27 (3), 259-269 (2018)

24. E. Grigut, I. Kiriyakov, Y.Senichenkov, ACM International Conference Proceeding Series, 17-22 (2018)

25. D. Japikse, E.M. Krivitzky, Proceedings of the ASME Turbo Expo (2016)

26. A.T. Lunev, PhD thesis, 123 (2005)

27. A.V. Semakov, V.E. Evdokimov, A.I. Reprintsev, A.N. Lyubimov Compr. Techn.and Pneum. 5, 2022 (2013)

28. A.N. Lyubimov, V.E. Evdokimov, A.V. Semakov and A.I. Reprintsev, Compr. Techn.and Pneum. 4, 12-20 (2014)

29. Y. Galerkin, K. Soldatova, Gas-dynamic process in industrial centrifugal compressors modeling. Scientific basis, steps of development, current state, 327 (2011)

30. Y.S. Vasilev, P.I. Rodionov, M.I. Socolovskiy Russ. Ind. 10-11, 78-85 (2000)

31. K.A. Danilov, PhD thesis, 176 (1999)

32. Y. Galerkin, K. Danilov, E. Popova IMechE Conference transactions "Compressors and their systems” (1999)

33. A.A. Drozdov, PhD thesis, 236 (2016)

34. V.F. Ris, Centrifugal compressor machines, 336 (1964)

35. Y. Galerkin, A. Rekstin, K. Soldatova, A. Drozdov, Compr. Techn.and Pneum. 6, 11-19 (2016)

36. K. Soldatova, Y. Galerkin, 18th International Conference on Mathematics and Statistical Engineering 3 (12), 822 (2016)

37. K. Soldatova, Dr. of tech. Sci. thesis, 257 (2017)

38. Yu.B. Galerkin, O.A. Solovyova, Compr. Techn. and Pneum. 3, 35-41 (2014)

39. Yu.B. Galerkin, O.A. Solovyova, Compr. Techn.and Pneum. 4, 15-21 (2014)

40. O.A. Solovyova, PhD thesis, 162 (2018)

41. G.N. Abramovich, Applied gas dynamics. Edition 4, amended and supplemented 888 (1976) 